\title{
ECTOPIC BEATS: HOW MANY COUNT?
}

\section{Rupert FG Simpson, ${ }^{1}$ Jessica Langtree, ${ }^{2}$ Andrew RJ Mitchell ${ }^{2}$}

\author{
1. King's College Hospital, London, UK \\ 2. Jersey General Hospital, Jersey, UK \\ *Correspondence to mail@jerseycardiologist.com
}

Disclosure: The authors have declared no conflicts of interest.

Received: 07.09.16 Accepted: 04.09.17

Citation: EMJ Cardiol. 2017;5[1]:88-92.

\begin{abstract}
Premature atrial and ventricular contractions, or ectopic beats, are frequently detected on routine electrocardiogram monitoring. They are often considered to be benign with no pathological significance; however, the literature suggests that higher ectopic burdens may have clinical importance. This paper reviews the current literature and provides the treating physician with an understanding of when ectopic beats should be deemed significant and when treatment may be appropriate.
\end{abstract}

Keywords: Premature atrial complex (PAC), premature ventricular complex (PVC), ectopy, ectopic beat, atrial fibrillation (AF), stroke.

\section{INTRODUCTION}

Premature atrial and ventricular contractions, or ectopic beats, are frequently detected on routine electrocardiogram (ECG) monitoring. They are often considered to be benign with no pathological significance; however, data suggest that higher ectopic burdens may have clinical importance.

\section{PREMATURE ATRIAL COMPLEXES}

Worldwide, stroke is a leading cause of mortality and the burden of disease on healthcare services is steadily increasing. ${ }^{1}$ Approximately $30-40 \%$ of ischaemic strokes are cryptogenic in nature with no clear pathological cause; ${ }^{2}$ it is thought that many could be secondary to subclinical or asymptomatic atrial fibrillation (AF). There is growing evidence that premature atrial complexes (PAC) may be associated with the development of AF, and therefore with an increased risk of stroke.

Haïssaguerre et al. ${ }^{3}$ investigated the link between PAC and the onset of AF. They identified individuals with frequent episodes of paroxysmal AF and mapped which rhythms commonly preceded the onset of AF. The trigger was often a PAC originating from a pulmonary vein, and radiofrequency ablation of this area of ectopic activity led to decreased recurrence in arrhythmic activity. ${ }^{3}$
Haïssaguerre et al.'s ${ }^{3}$ findings prompted further research toward the link between higher burdens of PAC and the risk of developing AF. Wallmann et al. ${ }^{4}$ recruited patients who had suffered an acute ischaemic stroke without prior documented AF and performed 7-day Holter monitoring at baseline, 3 months, and 6 months. Patients were then grouped according to their total burden of PAC over the 7-day period. A higher burden of PAC was classified as $\geq 70$ within the first 24-hour period. In individuals with a higher burden of PAC, $26 \%$ had $A F$, which was five-times more than those in the low burden group. ${ }^{4}$

The Copenhagen Holter study ${ }^{5}$ investigated AF prevalence and its effect on morbidity and mortality and was one of the largest studies of its kind in healthy individuals. Investigators contacted all men aged 55 years, and all men and women aged 65, 70, and 75 years in two different areas of the city. Individuals with previous cardiovascular ill health were excluded, leaving 678 participants who went on to complete 48-hour ambulatory ECG monitoring. In 2010, Binici et al. ${ }^{6}$ used these data to explore the link between excessive atrial ectopy and the primary endpoints of death or stroke, and secondary endpoint of AF. Excessive atrial ectopic activity was defined as $\geq 30$ PAC per hour or a single run of $\geq 20$. Over a median follow-up period of 76 months, it was found that excessive PAC 
were associated with a $>60 \%$ increase in the risk of death or stroke, and a 2.7-fold increase in the development of AF.

Using a more clinically applicable approach, Larsen et al. ${ }^{7}$ took data from the same study in an attempt to further clarify the link between higher burdens of atrial ectopy and stroke with a longer followup period (median: 14.4 years). Individuals were also risk stratified using the $\mathrm{CHA}_{2} \mathrm{DS}_{2}$ VASc scoring system (congestive heart failure, hypertension, aged $>75$ years, diabetes, previous stroke or transient ischaemic attack, vascular disease, aged 65-74 years, female sex). ${ }^{8}$ It was found that those with increased atrial ectopic activity had an increased adjusted risk of stroke (hazard ratio [HR]: 2.02; 95\% confidence interval [Cl]: 1.17-3.49), and a significantly higher risk of stroke $(p=0.0002)$ was identified in subjects with excessive PAC ( $\geq 30$ per hour or a run of $\geq 20$ ). Subjects with both excessive PAC and a $\mathrm{CHA}_{2} \mathrm{DS}_{2}$ VASc score $>2$ had an absolute risk of stroke equal to $2.4 \%$ per year. $^{7}$

The intervention arm of the EMBRACE trial ${ }^{9}$ was used to investigate the prevalence of subclinical $A F$ in patients who had suffered either a transient ischaemic attack or cryptogenic stroke. Recruits underwent 24-hour ambulatory ECG monitoring and, if AF was not detected on initial monitoring, they were then assigned to 30-day external loop recording. The overall 90-day AF detection rate was $16 \%$, and the probability of detecting AF increased with higher atrial ectopic activity. Patients with $<100 \mathrm{PAC} / 24$ hours had a probability of $A F$ detection of $<9 \%$, whereas the probability increased to $40 \%$ in those with a burden of $>1,500$ PAC/24 hours. ${ }^{9}$ This again serves to highlight the need for much longer periods of monitoring, especially in patients with higher PAC burdens.

\section{PREMATURE VENTRICULAR COMPLEXES}

Higher burdens of premature ventricular complexes (PVC) post myocardial infarction are associated with a poorer prognosis. ${ }^{10}$ Traditionally it has been thought that the use of anti-arrhythmic therapy in such patients may reduce the risk of sudden cardiac death; however, findings from CAST ${ }^{11}$ demonstrated that suppression of ventricular ectopy with Class 1a anti-arrhythmic agents was in fact associated with higher rates of death due to their proarrhythmic properties.

In patients with underlying structural heart disease, PVC can trigger ventricular arrhythmia, ${ }^{12}$ but in individuals with structurally normal hearts they are often considered a benign process that does not require treatment or intervention. ${ }^{13}$ However, Engel et al. $^{14}$ showed the presence of ventricular ectopics on resting ECG to be significant. Patients with resting ventricular ectopy had a significantly increased risk of all-cause and cardiovascular mortality. They also categorised patients by heart rate and showed mortality to increase with heart rate and to double in the presence of PVC. ${ }^{14}$ This increased adrenergic drive was proposed as a possible mechanism for incidental heart failure in tachycardia-driven PVC states.

The presence of PVC has been linked with incidental heart failure. In the ARIC study, Agarwal et al., ${ }^{15}$ found that participants, who at baseline had no heart failure or coronary artery disease, had an increased risk of incidental heart failure if PVC were present on baseline ECG. Further work by Agarwal et al. ${ }^{16}$ demonstrated that the presence of PVC was associated with nearly a two-fold risk of systolic heart failure.

A high frequency of PVC may result in left ventricular systolic dysfunction. In 2010, Baman et al. ${ }^{17}$ sought to quantify what burden of ventricular ectopy was associated with an increased risk of developing an ectopic-induced cardiomyopathy. ${ }^{17}$ They took patients with persistent ventricular ectopy despite best medical therapy, and calculated their PVC burden and left ventricular ejection fraction (LVEF) pre and post ablation. PVC-induced cardiomyopathy was defined as an improvement in LVEF of $\geq 15 \%$. The investigators identified that a total PVC burden of $>24 \%$ per 24 hours was associated with an increased risk of developing a cardiomyopathy with almost $80 \%$ sensitivity and specificity for the diagnosis of PVC-induced dilated cardiomyopathy. However, the minimal burden of PVC seen with cardiomyopathy is $10 \%$ over a 24 -hour period. ${ }^{17}$ The affect that a PVC has on ventricular filling and contractility, as well as the reversal in left ventricle systolic function seen post ablation, could make this a possible mechanism for left ventricle dysfunction in higher burdens of PVC. ${ }^{18}$

Penela et al. $^{19}$ further highlighted the importance of suppressing ventricular ectopy in patients with systolic function poor enough to mandate primary prevention implantable cardioverter defibrillator (ICD) implantation. Patients with high ectopic burdens who met the criteria for ICD implantation had the ICD withheld and instead underwent 
ventricular ectopic ablation. They were followed up at 6 and 12 months. The investigators found that at 12 months the LVEF had increased from a baseline of $28 \% \pm 4 \%$ to $42 \% \pm 12 \%$ at 12 months after PVC ablation. ${ }^{19}$ This emphasises the need for consideration of PVC ablation in those patients with indication for ICD and the potential for LVEF to recover, such that indication for ICD implantation can be reassessed at 12 months.

Dukes et al. ${ }^{20}$ further investigated the association between PVC burden and myocardial dysfunction. Participants with normal LVEF and no history of heart failure were studied; 1,139 were randomly assigned to 24-hour ambulatory ECG monitoring. Baseline echocardiography was performed and 842 participants went on to have repeat echocardiography after 5 years. Over the study period, it was shown that a two-fold increase in PVC burden from baseline was associated with a statistically significant greater chance of reduction in LVEF. Patients with known systolic dysfunction and higher burdens of ventricular ectopy showed a higher incidence of congestive cardiac failure (HR: 1.08; 95\% Cl: 1.03-1.17) and higher burdens of ventricular ectopy were also associated with increased mortality. ${ }^{20}$

The use of ambulatory monitors in many studies is reflected in clinical practice, as patients are often monitored for either 24 or 48 hours. Loring et al. ${ }^{21}$ demonstrated that $75 \%$ of patients that reach a PVC burden of $\geq 20 \%$ will do so within 24 hours of monitoring. However, only $53 \%$ of patients who reached a PVC burden of $10 \%$ did so in the same 24-hour timeframe. ${ }^{21}$ The yield continued to increase throughout the 14 days of monitoring; thus, leaving the possibility that almost half of this $10 \%$ PVC burden group may go undetected within a 24-hour monitoring period.

Increased ventricular ectopic activity is often seen during exercise stress testing; however, its clinical significance is poorly understood. Identifying the relationship between adverse outcomes and frequency of exercise-induced PVC has proven difficult. Schweikert et al. ${ }^{22}$ reported greater thallium perfusion defects with higher ectopic burdens, but these findings have not been shown to correspond with angiographic severity of disease. ${ }^{22}$ Other studies have suggested that whilst a causal link between exercise-induced ectopy and coronary artery disease does not exist, it may be a marker for increased risk of exerciseinduced ventricular arrhythmia. ${ }^{23}$
Jouven et al. ${ }^{24}$ performed exercise testing in 6,106 asymptomatic male volunteers and measured ventricular ectopic burden. Excessive ventricular ectopic activity was defined as $>10 \%$ of all ventricular depolarisations during a 30-second ECG recording or a run of $\geq 2$ consecutive PVC. Frequent ventricular ectopy was identified in 138 participants and, over a 23-year follow-up, was associated with an increased risk of death from a cardiovascular cause (relative risk: $2.67 ; 95 \% \mathrm{Cl}$ : 1.76-4.07).

Frolkis et al. ${ }^{25}$ retrospectively examined a large cohort of 29,244 patients who had previously undergone exercise testing. Frequent ventricular ectopy was defined as $>7$ PVC per minute, or the presence of bigeminy, trigeminy, ventricular tachycardia, or fibrillation. Frequent ventricular ectopy was identified in $3 \%$ of individuals during exercise and $2 \%$ during recovery. Over a mean follow-up of 5.3 years, frequent ventricular ectopic activity in recovery was associated with a higher rate of death when compared with ectopy seen on exercising (11\% versus 5\%; HR: 2.4; 95\% Cl: 2.0-2.9; $\mathrm{p}<0.001)$. An assessment of LVEF had been made on 6,421 participants and a higher proportion of patients with ventricular ectopy during recovery had a LVEF of $<40 \%$ ( $27 \%$ versus $18 \%$ ).

Morshedi-Meibodi et al. ${ }^{26}$ retrospectively examined ventricular ectopic activity in 2,885 individuals who had undergone exercise testing as part of the Framingham offspring study. Their definition of excessive ventricular ectopic burden differed from Jouven et al.'s ${ }^{24}$ as only $0.1 \%$ of participants would have met the required standard. They instead used a model based on the median number of ventricular ectopics measured in participants whilst exercising, which was one ectopic every 4.5 minutes (0.22 ectopics/minute), and excessive activity was seen in 792 (27\%) participants. Their primary endpoint was 'hard' cardiovascular disease, which included angina symptoms, myocardial infarction, and sudden cardiac death. It was concluded that there was no association between high PVC burden and any of the 'hard' cardiovascular disease endpoints; however, there was an increase in allcause mortality over a follow-up period of 15 years.

\section{DISCUSSION}

AF and atrial flutter are the most common arrhythmias associated with ischaemic stroke. There is now growing evidence supporting an association between atrial ectopic activity and the development of atrial arrhythmias. Individuals with 
high atrial ectopic burdens are at a greater risk of stroke and death, possibly due to co-existing AF. Patients with $\mathrm{AF}$ and a $\mathrm{CHA}_{2} \mathrm{DS}_{2}$ VASc score of two have an annual stroke risk of $2.2 \%$ and should be appropriately anticoagulated unless contraindicated. Data presented in this review suggest that individuals with a high PAC burden of $>30$ per hour and $\mathrm{CHA}_{2} \mathrm{DS}_{2}$ VASc score of two also have an increased risk of stroke. Randomised trials are needed to assess whether anticoagulation (and the inherent risks that come with it), suppression of atrial ectopics, or modification of other risk factors decrease stroke risk in patients with high PAC burden. Clinical suspicion of undiagnosed AF should be higher in patients with a high $\mathrm{CHA}_{2} \mathrm{DS}_{2}$ VASc score, an atrial ectopic burden of $\geq 30 /$ hour, or an episode of $\geq 20$ PAC. More extended periods of ambulatory monitoring, including implantation of loop recorders, may increase the chance of diagnosing asymptomatic $\mathrm{AF}^{27}$

As many as $50 \%$ of cases of congestive cardiac failure are labelled as idiopathic, yet many may be secondary to excessive ventricular ectopic activity. $^{28}$ In a meta-analysis, Zang et al. ${ }^{29}$ demonstrated an improvement in LVEF following ablation of PVC. The mean burden of PVC referred for an ablation was $24.0 \%$ and the overall increase in LVEF post ablation was $7.7 \% .{ }^{29}$ Patients with high PVC burdens should therefore be considered for regular echocardiographic assessment and those who show deterioration in LVEF should be assessed for treatment with catheter ablation. The significance of ectopic burden during exercise testing, or in the recovery period, is as of yet unknown. Data does suggest a link to all-cause mortality over long follow-up periods, but there is no current evidence to suggest that suppression of PVC in this setting would be beneficial. As such, catheter ablation of PVC in this setting is an untested area and, in symptomatic patients, clinicians may first wish to employ a less invasive approach, such as anti-arrhythmic therapy.

\section{CONCLUSION}

Higher burdens of PAC are associated with a greater risk of developing AF. High frequency of PVC is associated with a reduction in LVEF and subsequent heart failure, which may be reversible with ablation. In all cases, longer periods of monitoring allow a greater yield of information and better prediction of those higher risk patients. Longer periods of monitoring of $\leq 2$ weeks should become commonplace.

\section{REFERENCES}

1. Feigin VL et al. Global and regional burden of stroke during 1990-2010: findings from the Global Burden of Disease Study 2010. Lancet. 2014; 383(9913):245-54.

2. Guercini $F$ et al. Cryptogenic stroke: time to determine aetiology. J Thromb Haemost. 2008;6(4):549-54.

3. Haïssaguerre $M$ et al. Spontaneous initiation of atrial fibrillation by ectopic beats originating in the pulmonary veins. N Engl J Med. 1998;339(10):659-66.

4. Wallmann $D$ et al. Frequent atrial premature beats predict paroxysmal atrial fibrillation in stroke patients: an opportunity for a new diagnostic strategy. Stroke. 2007;38(8):2292-4.

5. Sajadieh A et al. Prevalence and prognostic significance of daily-life silent myocardial ischaemia in middle-aged and elderly subjects with no apparent heart disease. Eur Heart J. 2005;26(14):1402-9.

6. Binici Z et al. Excessive supraventricular ectopic activity and increased risk of atrial fibrillation and stroke. Circulation. 2010;121(17):1904-11.

7. Larsen BS et al. Excessive atrial ectopy and short atrial runs increase the risk of stroke beyond incident atrial fibrillation. J Am Coll Cardiol. 2015;66(3):232-41.

8. Lip GY et al. Refining clinical risk stratification for predicting stroke and thromboembolism in atrial fibrillation using a novel risk factor-based approach: the euro heart survey on atrial fibrillation. Chest. 2010;137(2):263-72.

9. Gladstone DJ et al. Atrial premature beats predict atrial fibrillation in cryptogenic stroke: results from the EMBRACE trial. Stroke. 2015;46(4): 936-41.

10. Kostis JB et al. Prognostic significance of ventricular ectopic activity in survivors of acute myocardial infarction. J Am Coll Cardiol. 1987;10(2):231-42.

11. Echt DS et al. Mortality and morbidity in patients receiving encainide, flecainide, or placebo. The Cardiac Arrhythmia Suppression Trial. N Engl J Med. 1991; 324(12):781-8.

12. Myerburg RJ et al. Sudden cardiac death: epidemiology, transient risk, and intervention assessment. Ann Intern Med. 1993;119(12):1187-97.
13. Conti CR. Ventricular arrhythmias: A general cardiologist's assessment of therapies in 2005. Clin Cardiol. 2005; 28(7):314-6.

14. Engel $G$ et al. Prognostic significance of PVCs and resting heart rate. Ann Noninvasive Electrocardiol. 2007;12(2): 121-9.

15. Agarwal SK et al. Relationship of ventricular premature complexes to heart failure (from the atherosclerosis risk in communities [ARIC] study). Am J Cardiol. 2012;109(1):105-9.

16. Agarwal $\vee$ et al. Relation between ventricular premature complexes and incident heart failure. Am J Cardiol. 2017; 119 (8):1238-42.

17. Baman TS et al. Relationship between burden of premature ventricular complexes and left ventricular function. Heart Rhythm. 2010;7(7):865-9.

18. Malik M, Cam JA. "Dynamic electrocardiography," Malik M, Cam JA (eds.), (2004), Elmsford, N.Y.: Blackwell Futura.

19. Penela $D$ et al. Ablation of frequent PVC in patients meeting criteria for 
primary prevention ICD implant: Safety of withholding the implant. Heart Rhythm. 2015;12(12):2434-42.

20. Dukes JW et al. Ventricular ectopy as a predictor of heart failure and death. $J$ Am Coll Cardiol. 2015;66(2):101-9.

21. Loring $Z$ et al. Longer ambulatory ECG monitoring increases identification of clinically significant ectopy. Pacing Clin Electrophysiol. 2016;39(6):592-7.

22. Schweikert RA et al. Association of exercise-induced ventricular ectopic activity with thallium myocardial perfusion and angiographic coronary artery disease in stable, low-risk populations. Am J Cardiol. 1999;83(4): 530-4.
23. Partington $S$ et al. Prevalence and prognostic value of exercise-induced ventricular arrhythmias. Am Heart J. 2003;145(1):139-46.

24. Jouven $X$ et al. Long-term outcome in asymptomatic men with exercise-induced premature ventricular depolarizations. N Engl J Med. 2000;343(12):826-33.

25. Frolkis JP et al. Frequent ventricular ectopy after exercise as a predictor of death. N Engl J Med. 2003;348(9):781-90.

26. Morshedi-Meibodi A et al. Clinical correlates and prognostic significance of exercise-induced ventricular premature beats in the community: the Framingham Heart Study. Circulation. 2004;109(20):2417-22.
27. Kernan WN et al. Guidelines for the prevention of stroke in patients with stroke and transient ischemic attack: A guideline for healthcare professionals from the American Heart Association/ American Stroke Association. Stroke. 2014;45(7):2160-236.

28. Felker GM et al. Underlying causes and long-term survival in patients with initially unexplained cardiomyopathy. N Engl J Med. 2000;342(15):1077-84.

29. Zang $M$ et al. Beneficial effects of catheter ablation of frequent premature ventricular complexes on left ventricular function. Heart. 2014;100(10):787-93. 